\title{
Finite element and finite volume-element simulation of pseudo-ECGs and cardiac alternans
}

\author{
Marie Dupraz ${ }^{a}$, Simonetta Filippi ${ }^{b}$, Alessio Gizzi ${ }^{b}$, Alfio Quarteroni ${ }^{a, c}$ and \\ Ricardo Ruiz-Baier ${ }^{\mathrm{d} * \dagger}$
}

\section{Communicated by S. Wise}

In this paper, we are interested in the spatio-temporal dynamics of the transmembrane potential in paced isotropic and anisotropic cardiac tissues. In particular, we observe a specific precursor of cardiac arrhythmias that is the presence of alternans in the action potential duration. The underlying mathematical model consists of a reaction-diffusion system describing the propagation of the electric potential and the nonlinear interaction with ionic gating variables. Either conforming piecewise continuous finite elements or a finite volume-element scheme are employed for the spatial discretization of all fields, whereas operator splitting strategies of first and second order are used for the time integration. We also describe an efficient mechanism to compute pseudo-ECG signals, and we analyze restitution curves and alternans patterns for physiological and pathological cardiac rhythms. Copyright @ 2014 John Wiley \& Sons, Ltd.

Keywords: finite element discretization; finite volume-element method; reaction-diffusion system; operator splitting; cardiac alternans; pseudo-ECG; spatio-temporal dynamics

\section{Introduction}

Cardiac arrhythmias represent one of the major causes of morbidity and mortality in industrialized countries. They are associated with important risk factors and comorbidities as hypertension, diabetes, and other aging pathologies, in particular with strokes. Arrhythmias represent, in fact, a pathology included in the priority investigation lines of the European research program HORIZON 2020. International strategies have been oriented toward the development of new and more efficient methods and devices of prevention, as well as medical care in order to face the growing costs connected with an increasing life expectancy. The study of such a complex phenomenon requires, therefore, a Systems Biology multidisciplinary and integrative approach, where clinical and experimental investigations are integrated by in silico analysis.

It is well known that heart tissue is a complex biological excitable media that supports rotating activation waves [1,2] which induce regular and irregular rhythms leading to life threatening arrhythmias. Spiral (two dimensional) and scroll (three dimensional) waves have been experimentally observed and theoretically predicted during tachycardia and out-of phase stimuli. They are recognized to be responsible for fibrillation scenarios $[3,4]$ both in normal and altered thermal conditions $[5,6]$.

Based on these considerations, the mathematical modeling of cardiac electrical activity has received a great deal of interest in the last few decades [7]. On the lines of the pioneering work of Hodgkin-Huxley, their experimental methodology and theoretical formalism [8], a variety of mathematical models have been introduced to capture some important features of this complex phenomenon, typically regarding the heart as an excitable medium, therefore studying the underlying mechanisms within the framework of reaction-diffusion systems (see, e.g., [9]). The quantity and quality of such studies has been also boosted by the increasing capacity of modern computers and numerical techniques (see, e.g., [10]). Virtually, all major types of numerical methods have been successfully employed to simulate

\footnotetext{
a Modeling and Scientific Computing, MATHICSE, École Polytechnique Fédérale de Lausanne, CH-1015 Lausanne, Switzerland

${ }^{b}$ Nonlinear Physics and Mathematical Modeling Lab, University Campus Bio-Medico of Rome, I-00128 Rome, Italy

c MOX - Politecnico di Milano, piazza Leonardo da Vinci 32, 20133 Milano, Italy

$d$ Institute of Earth Sciences, FGSE, University of Lausanne, CH-1015 Lausanne, Switzerland

* Correspondence to: Ricardo Ruiz-Baier, Institute of Earth Sciences, FGSE, University of Lausanne, CH-1015 Lausanne, Switzerland.

†E-mail: ricardo.ruizbaier@unil.ch
} 
the so-called monodomain or bidomain equations in 3D geometries, including finite differences [11, 12], finite volume formulations $[13,14]$, finite elements (FEs) [15-19], and spectral discretizations [20].

In this work, we focus on the monodomain mathematical formalism to study emerging spatio-temporal dynamics and alternans behavior in two-dimensional and three-dimensional domains. The underlying system of equations consists of a parabolic PDE describing the propagation of membrane depolarization, nonlinearly coupled with a set of ODEs governing the dynamics of gating variables, and ionic concentrations. The membrane kinetics are modeled adopting the four-variable minimal phenomenological model for cardiac action potential propagation from [21]. Despite the current level of ionic models complexity (state-of-the-art models may include more than sixty variables, see, e.g., [22]), the minimal model allows us to reproduce key experimental-based cardiac action potential restitution properties and to investigate pro-arrhythmic states with comparable accuracy to more sophisticated ionic models.

In the present work, we further introduce dedicate numerical solvers based on piecewise linear FEs and operator splitting strategies for the space-time discretization. Differently from other computational work in cardiac electrophysiology, these schemes, under some particular conditions, are able to provide accurate and efficient numerical approximations. In addition, we introduce a finite volumeelement (FVE) method, where one of the main goals is to improve the mass conservation properties of the overall discretization. FVE methods are nonstandard hybrid discretizations that combine some features of classical FE methods and finite volume schemes, such as smooth derivability of error estimates in natural norms and local conservativity of fluxes. We recall that the main idea is to write a finite volume method on an adjoint mesh and to use a piecewise constant interpolation operator to recast the formulation as a PetrovGalerkin scheme. Different flavors of these methods have been typically applied in the simulation of flow processes [23-28], and up to our best knowledge, the present paper is the first one addressing FVE discretizations specifically tailored for cardiac-related problems. We point out that a somewhat related method has also been applied to cardiac electrophysiology in [29]; however, our method is more closely linked to the formulations introduced in [30-33] for reaction-diffusion systems. Another goal of this paper is to quantitatively assess the accuracy of the proposed numerical schemes in terms of correct reproduction of several electrophysiological scenarios of interest in cardiac modeling.

The phenomenon of alternating variations in the amplitude of, for example, membrane action potential waves over successive cardiac cycles is commonly known as cardiac alternans (see, e.g., [34-36]). Our extended numerical study in two and three dimensions allows us to characterize the behavior of these alternans in terms of reconstructed ECG signals (called pseudo-ECG [60]), conduction velocity (CV), and action potential duration (APD) restitution curves [37], in the framework of the so-called forward problem in electrocardiology. Such a complex topic exhibits several unknowns both from the experimental and theoretical points of view. Moreover, different numerical results are still debated in terms of which mathematical models are able to correctly reproduce the spatio-temporal onset and transition of alternans patterns toward ventricular fibrillation. In this work, we show the ability of the proposed numerical schemes to accurately render space and time alternans patterns and discuss and analyze in detail the tissue-dependent properties (i.e., myocardial fiber rotational anisotropy [38]) of the studied phenomena.

The remainder of this paper is organized as follows. Section 2 outlines the mathematical model of cardiac electrophysiology including the monodomain equations, the choice of ionic model, and presents the main elements of ECG modeling and observation of alternans. We describe in Section 3 the FE and FVE discretizations along with the operator splitting employed in our numerical framework. A set of illustrative examples is collected in Section 4, and we close with some remarks and discussion in Section 5.

\section{Governing equations, pseudo-ECG modeling, and alternans dynamics}

\subsection{The monodomain model}

Let $\Omega \subset \mathbb{R}^{3}$ denote a domain with smooth boundary $\partial \Omega$ and consider a finite time interval $(0, T)$ for a fixed $T>0$. The so-called monodomain equation (also known as cable equation) results from the Kirchhoff's current law, and it addresses single cell excitation, that is, depolarization and repolarization dynamics, describing the propagation of the transmembrane potential through the tissue via a diffusive Fourier formalism [39]. It consists in the following reaction-diffusion system where the unknown quantities are the transmembrane potential $v=v(x, t)$ and a vector field of $s$ ionic variables (named gating variables) $\boldsymbol{i}=\boldsymbol{i}(x, t)$ :

$$
\begin{aligned}
C_{m} \chi^{-1} \partial_{t} v-\nabla \cdot(\sigma \nabla v)+\chi^{-1} \mathbf{I}_{\text {ion }}(v, i) & =\mathbf{I}_{\text {app }} \quad \text { in } \Omega \times(0, T], \\
\mathrm{d}_{t} \boldsymbol{i}-\boldsymbol{m}(\boldsymbol{v}, i) & =0 \quad \text { in } \Omega \times(0, T] .
\end{aligned}
$$

Here, $C_{m}$ is the membrane capacitance per unit area, $\chi$ is the surface-to-volume ratio of the myocardium, $\mathbf{I}_{\text {ion }}$ is the sum of the ionic currents, $\mathbf{I}_{\mathrm{app}}$ is an extracellular current density stimulus, and $\sigma=\sigma_{f} \mathbf{I}+\left(\sigma_{s}-\sigma_{f}\right) \mathbf{f}_{0} \otimes \mathbf{f}_{0}$ is a transversely isotropic conductivity tensor representing different myocardial propagation velocities $\sigma_{f}, \sigma_{s}$ in the parallel and perpendicular myofiber directions, $\mathbf{f}_{0}, \mathbf{s}_{0}$, respectively (see, e.g., [17]). As usual, the symbols $\partial_{t}, d_{t}, \nabla$, and $\nabla$. represent the partial and total derivatives with respect to time $t$, spatial gradient and divergence operators, respectively.

The cardiac muscle consists of densely packed cells organized in such a way that the mean electric conductivity is much higher in the direction of the fibers $\mathbf{f}_{0}$. In Cartesian coordinates, we have

$$
\boldsymbol{\sigma}=\left(\begin{array}{ccc}
\sigma_{f} \cos ^{2} \theta(z)+\sigma_{s} \sin ^{2} \theta(z) & \left(\sigma_{f}-\sigma_{s}\right) \sin \theta(z) \cos \theta(z) & 0 \\
\left(\sigma_{f}-\sigma_{s}\right) \sin \theta(z) \cos \theta(z) & \sigma_{f} \sin ^{2} \theta(z)+\sigma_{s} \cos ^{2} \theta(z) & 0 \\
0 & 0 & \sigma_{s}
\end{array}\right)
$$

where $\theta(z)$ denotes the angle between the fiber and the $y$-axis in each plane (Figure 1). 

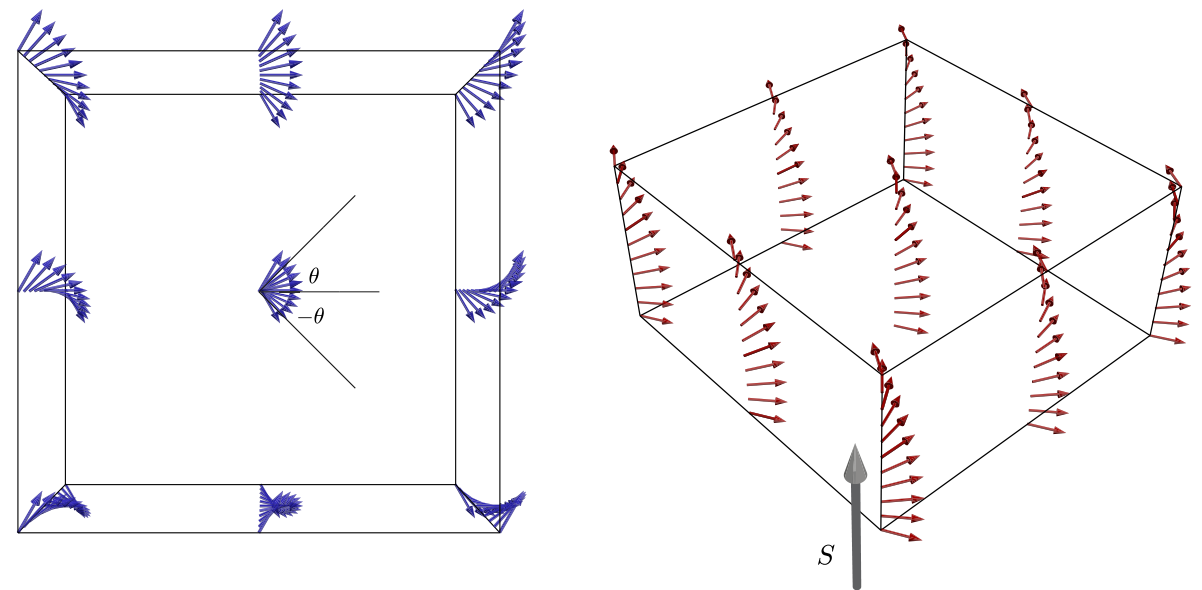

Figure 1. Sketch of the 3D slab of cardiac tissue employed in the simulations. The fibers vary transmurally from $60^{\circ}$ at $z=1$ to $-60^{\circ}$ on $z=0$. Such conditions are representative of the left ventricle wall.

System (2.1) is complemented with zero-flux boundary conditions for the transmembrane potential $[\boldsymbol{\sigma}(x) \nabla v] \cdot \boldsymbol{n}=0$ on $\partial \Omega$, representing that the tissue is isolated, and suitable initial potential and ionic distributions $v(x, 0)=v_{0}, i(x, 0)=i^{0}$ are identified.

The specification of the reaction terms $\mathbf{I}_{\text {ion }}$ and $\boldsymbol{m}$ determines, respectively, the ionic currents across the cell membrane and concentration of ionic quantities, which will be considered in the model. Depending on the target application, cell models with different levels of physiological detail can be incorporated, ranging from phenomenological (which reproduce the shape and structure of the action potential) to physiologically accurate descriptions of the microscopic subcellular dynamics (see a mathematical analysis of a class of reaction-diffusion systems governing membrane dynamics in, e.g., [40]). Here, we focus on the phenomenological minimal model for human species, introduced in [21]. This four-field model is capable to accurately recover several physiological and pathological conditions. These include, for instance, resting potential and excitation thresholds, shape-duration of the action potential and restitution dynamics, CV, and wave break. The kinetics are specified as

$$
\begin{aligned}
& \mathbf{I}_{\text {ion }}(v, i)=-i_{1} H\left(v-\theta_{1}\right)\left(v-\theta_{1}\right)\left(v_{v}-v\right) / \tau_{f}+\left(v-v_{0}\right)\left(1-H\left(v-\theta_{2}\right)\right) / \tau_{0}+H\left(v-\theta_{2}\right) / \tau_{3,0}-H\left(v-\theta_{2}\right) i_{2} i_{3} / \tau_{s i}, \\
& m(v, i)=\left(\begin{array}{c}
\left(\left(1-H\left(v-\theta_{1}\right)\right)\left(i_{1, \text { inf }}-i_{1}\right) / \tau_{1}^{-}-H\left(v-\theta_{1}\right) i_{1} / \tau_{1}^{+}\right. \\
\left(1-H\left(v-\theta_{2}\right)\right)\left(i_{2, \text { inf }}-i_{2}\right) / \tau_{2}^{-}-H\left(v-\theta_{2}\right) i_{2} / \tau_{2}^{+} \\
\left.\left(\left(1+\tanh \left(k_{3}\left(v-v_{3}\right)\right)\right) / 2-i_{3}\right) / \tau_{3}\right)^{T}
\end{array}\right),
\end{aligned}
$$

where $H$ is the Heaviside function while switches and infinite values are defined as

$$
\begin{aligned}
\tau_{1}^{-} & =\left(\left(1-H\left(v-\theta_{1}^{-}\right) v\right) \tau_{1,1}^{-}+H\left(v-\theta_{1}^{-}\right) \tau_{1,2}^{-},\right. \\
\tau_{2}^{-} & =\tau_{2,1}^{-}+\left(\tau_{2,2}^{-}-\tau_{2,1}^{-}\right)\left(1+\tanh \left(k_{2}^{-}\left(v-v_{2}^{-}\right)\right)\right) / 2, \\
\tau_{3,0}= & \tau_{3,0,1}+\left(\tau_{3,0,2}-\tau_{3,0,1}\right)\left(1+\tanh \left(k_{3,0}\left(v-v_{3,0}\right)\right)\right) / 2, \\
\tau_{3}= & \left(\left(1-H\left(v-\theta_{2}\right)\right) \tau_{3,1}+H\left(v-\theta_{2}\right) \tau_{3,2},\right. \\
\tau_{0}= & \left(\left(1-H\left(v-\theta_{0}\right)\right) \tau_{0,1}+H\left(v-\theta_{0}\right) \tau_{0,2},\right. \\
i_{1, \text { inf }}= & 1, v<\theta_{1}^{-} \\
i_{2, \text { inf }}= & \left(\left(1-H\left(v-\theta_{1}^{-},\right.\right.\right.
\end{aligned}
$$

All parameter values are specified in Table I.

\subsection{Pseudo-ECG representation}

Electrocardiogram is the most used device to record the external potential at the torso surface. These data are employed to assess the properties of the electrical activity in the heart. To recover this signal, one could solve a full heart-torso coupled problem (see, e.g., $[41,42]$ ), or alternatively, obtain an approximation (which is, of course, less accurate) called pseudo-ECG. Basically, the latter consists in averaging the potential propagation through the torso recorded at a given electrode $e$. Let $x_{e}$ denote the position of $e$ at the torso surface (that is, $x_{e} \notin \bar{\Omega}$ ). Assuming that the extra-myocardial matrix and the torso possess an isotropic conductivity $\sigma=\sigma \mathbf{l}$, for a given $\sigma>0$, and assuming that impressed density current is $\sigma \nabla v$, we can write the following approximation for the shape of the ECG at $x_{e}$

$$
\operatorname{ECG}\left(x_{e}\right):=\int_{\Omega} \frac{\Delta v(x, t)}{\left\|x-x_{e}\right\|} d x
$$


Table I. Typical values for model and discretization parameters.

Parameters

lonic cell model parameters

Monodomain model parameters Discretization and pacing parameters

$$
\begin{aligned}
& \theta_{0}=0.005, \theta_{1}=0.3, \theta_{2}=0.13, \theta_{1}^{-}=0.1, \tau_{3,0,1}=91, \tau_{3,0,2}=0.8, \tau_{3,1}=2.7342, \tau_{3,2}=4, \\
& \tau_{0,1}=410, \tau_{0,2}=7, i_{2, \infty}^{*}=0.5, v_{v}=1.61, \tau_{1,1}^{-}=80, \tau_{1,2}^{-}=\tau_{1}^{+}=1.4506, \tau_{2,1}^{-}=70, \\
& \tau_{2,2}^{-}=8, \tau_{2}^{+}=280, \\
& k_{2}^{-}=200, v_{2}^{-}=0.016, \tau_{f}=0.078, k_{3,0}=2.1, v_{3,0}=0.6, k_{3}=2.0994, v_{3}=0.9087, \\
& \tau_{s i}=3.3849, \tau_{2, \infty}=0.01 \\
& C_{m}=1 \mu \mathrm{F} / \mathrm{cm}^{2}, \chi=1400 \mathrm{~cm}, \sigma_{f}=0.001 \mathrm{~cm}^{2} / \mathrm{ms}, \sigma_{s}=0.0002 \mathrm{~cm}^{2} / \mathrm{ms} \\
& \Omega=(0,1) \times(0,1) \times(0,0.0625) \mathrm{cm}^{3}, h=0.005 \mathrm{~cm}, \Delta t=0.01 \mathrm{~ms}, \ell=5, \delta t=5 \mathrm{~ms}, \eta=2 \mathrm{~ms}
\end{aligned}
$$

The derivation of (2.2) relies on Ohm's law, Green's formula, and the relations between a potential, currents, and a point source [43]. Such an approximation therefore becomes useful to get further information concerning the electrical activity of the heart, specially when in vivo action potential tissue measurements are not feasible.

\subsection{Dynamics of cardiac alternans}

Alternans of APD at the cellular and tissue levels is usually associated with alternans of the T-wave in the ECG signal [44-46], that is, there exists an alternating variation in the amplitude or shape of the T-wave. With such an expression, it is possible to identify a condition that rises during fast pacing of the tissue, resulting in a regular and stable alternations of the action potential between higher-longer and smaller-shorter waves, in time. Although the mechanisms for alternating signals have been theoretically explored in zero-dimensional settings (see, e.g., [34-36]), only a few experimental and theoretical evidences have been discussed in higher spatial dimensions [47-52]. Both in-phase (concordant) and out-of-phase (discordant) patterns have been recently shown to arise because of several different and cumulative effects [53,54], encompassing:

(i) concordant phases can develop from discordant alternans as the pacing period is decreased;

(ii) multiple stationary nodal lines may exist, as alternans exhibit full three-dimensional dynamics;

(iii) the complex spatio-temporal alternans patterns are very sensitive to both the stimulation site and simulation history. These evidences indicate the hidden predisposition of such a behavior to chaotic regimes and how little is our understanding of the phenomenon.

The most adopted alternans analysis is based on the study of the so-called restitution curve of the APD (Section 4). According to onedimensional map theory, Nolasco and Dahlen [37] defined a criterion for alternans onset based on the slope of such a curve. In this work, we make use of such a criterion, but we further analyze the resulting spatio-temporal dynamics by adopting the following definition of alternans at a generic location in the Cartesian space:

$$
\Delta \operatorname{APD}(x, y, z)_{n}=\operatorname{APD}(x, y, z)_{n+1}-\operatorname{APD}(x, y, z)_{n} \rightarrow \begin{cases}\left|\Delta \operatorname{APD}(x, y, z)_{n}\right|>\eta & \text { Alternans } \\ \left|\Delta \operatorname{APD}(x, y, z)_{n}\right| \leq \eta & \text { Nodal line }\end{cases}
$$

In particular, we compute the difference between two consecutive APDs, at pacing $n$ and $n+1$, thus comparing the absolute difference with a reference threshold $\eta=2 \mathrm{~ms}$, we distinguish between non alternating (Nodal line) and alternating (Alternans) regions. Such a procedure allows us to determine the temporal distribution of action potential alternans across the domain. In fact, by plotting the actual difference $\triangle \operatorname{APD}(x, y, z)_{n}$ in the mapped field, we can visualize in-phase and out-of-phase regions (Figure 7).

\section{Discretization}

\subsection{Finite element setting}

The spatial domain $\Omega$ is discretized into tetrahedral elements $K$ of diameter $h_{K}$ providing a conforming partition $\mathcal{T}_{h}$ of meshsize $h:=$ max $h_{K}$ consisting of $N_{h}$ vertexes. By $\mathbb{P}_{1}(K)$, we denote the space of affine functions defined in $K$. The classical finite dimensional space of continuous piecewise linear functions is (see, e.g., [55])

$$
V_{h}=\left\{v_{h} \in C^{0}(\bar{\Omega}):\left.v_{h}\right|_{K} \in \mathbb{P}_{1}(K), \forall K \in \mathcal{T}_{h}\right\}
$$

and it is spanned by a set of Lagrangian basis functions $\left\{\psi_{i}\right\}_{i=1}^{N_{h}}$. The spatial semidiscrete Galerkin formulation for (2.1) consists in finding $v_{h} \in V_{h}, i_{h} \in\left[V_{h}\right]^{s}$ such that for each $t \in(0, T)$

$$
\begin{aligned}
& C_{m} \chi^{-1} \int_{\Omega} \partial_{t} v_{h}(t) w_{h} d x+\int_{\Omega} \sigma \nabla v_{h}(t) \cdot \nabla w_{h} d x+\chi^{-1} \int_{\Omega} \mathbf{I}_{\text {ion }}\left(v_{h}(t), i_{h}(t)\right) w_{h} d x=\int_{\Omega} \mathbf{I}_{\text {app }} w_{h} d x \quad \forall w_{h} \in V_{h}, \\
& \int_{\Omega} \mathrm{d}_{t} \boldsymbol{i}_{h}(t) \cdot \boldsymbol{j}_{h} d x-\int_{\Omega} m\left(v_{h}(t), \boldsymbol{i}_{h}(t)\right) \cdot \boldsymbol{j}_{h} d x=0 \quad \forall \boldsymbol{j}_{h} \in\left[V_{h}\right]^{s} .
\end{aligned}
$$


Let us denote mass and stiffness matrices respectively by $\mathbf{M}, \mathbf{A}$, with components

$$
m_{k l}:=\sum_{K \in \mathcal{T}_{h}} \int_{K} \psi_{k} \psi_{l} d x, \quad a_{k l}:=\sum_{K \in \mathcal{T}_{h}} \int_{K} \sigma \nabla \psi_{k} \cdot \nabla \psi_{l} d x
$$

and define the vectors $\mathbf{V}=(v)_{j}, \mathbf{J}=(i)_{j}, \mathbf{I}_{\text {ion }}=\left(\mathbf{I}_{\text {ion }}\right)_{j}, \mathbf{I}_{\text {app }}=\left(\mathbf{I}_{\text {app }}\right)_{j}, j=1, \ldots, N_{h}$, where the subindices denote that the quantity corresponds to the node $j$ of $\mathcal{T}_{h}$. Then the semidiscrete FE approximation of the monodomain reaction-diffusion model reads

$$
\begin{aligned}
C_{m} \chi^{-1} \mathbf{M} \partial_{t} \mathbf{V}-\mathbf{A} \mathbf{V}+\chi^{-1} \mathbf{M} \mathbf{I}_{\text {ion }} & =\mathbf{M} \mathbf{I}_{\mathrm{app}}, \\
\mathrm{d}_{t} \mathbf{J} & =m(\mathbf{V}, \mathbf{J}) .
\end{aligned}
$$

\subsection{Finite volume-element formulation}

Let $\mathcal{T}_{h}^{*}$ be a dual partition of $\Omega$ into finite volumes (here polygons) $K_{j}^{*}$ centered on each node $x_{j}, j=1, \ldots, N_{h}$ of the primal mesh $\mathcal{T}_{h}$. Let $b_{K}$ denote the barycenter of a generic element $K \in \mathcal{T}_{h}$. Dual elements are constructed by joining the barycenter $b_{K}$ of each primal element $K$ sharing the vertex $x_{j}$, with the midpoints of the faces intersecting $x_{j}$. A finite dimensional space associated to piecewise constant functions defined on this dual mesh is defined as follows:

$$
V_{h}^{*}:=\left\{v_{h} \in L^{2}(\Omega):\left.v_{h}\right|_{K_{j}^{*}} \in \mathbb{P}^{0}\left(K_{j}^{*}\right), \forall K_{j}^{*} \in \mathcal{T}_{h}^{*}\right\}
$$

and a connection between $V_{h}$ and $V_{h}^{\star}$ is defined by the piecewise constant interpolator $\pi_{h}: V_{h} \rightarrow V_{h}^{*}$ (cf., e.g., [32]) specifically defined by

$$
\left(\pi_{h} v_{h}\right)(x)=\sum_{j=1}^{N_{h}} v_{h}\left(x_{j}\right) \chi_{j}(x) \quad \text { for } x \in \Omega,
$$

where $\chi_{j}$ stands for the characteristic function on the control volume $K_{j}^{\star} \in \mathcal{T}_{h}^{*}$. Its vectorial counterpart is denoted by $\Pi_{h}:\left[V_{h}\right]^{s} \rightarrow$ $\left[V_{h}^{*}\right]^{s}, s \geq 1$. Let $w_{h} \in V_{h}, j_{h} \in\left[V_{h}\right]^{s}$. Multiplication of the first and second equations in (2.1) by the test functions $\pi_{h} w_{h} \in V_{h}^{\star}, \Pi_{h} j_{h} \in$ $\left[V_{h}^{*}\right]^{s}$ (respectively), integration by parts over each control volume $K_{j}^{*} \in \mathcal{T}_{h}^{*}$ and summation yields a semidiscrete finite volume method written in the form

$$
\begin{gathered}
\chi^{-1} \sum_{K_{j}^{*} \in \mathcal{T}_{h}^{*}}\left(C_{m} \partial_{t} v_{h}(t)+\left(\mathbf{I}_{\text {ion }}\left(v_{h}(t), \boldsymbol{i}_{h}(t)\right), \pi_{h} w_{h}\right)-\sum_{K_{j}^{*} \in \mathcal{T}_{h}^{*}} \int_{K_{j}^{*} \in \mathcal{T}_{h}^{*}}\left(\sigma \nabla v_{h}(t) \cdot \boldsymbol{n}\right) \pi_{h} w_{h} d s=\sum_{K_{j}^{*} \in \mathcal{T}_{h}^{*}}\left(\mathbf{I}_{\mathrm{app}}, \pi_{h} w_{h}\right),\right. \\
\sum_{K_{j}^{*} \in \mathcal{T}_{h}^{*}}\left(\mathrm{~d}_{t} \boldsymbol{i}_{h}(t)-m\left(v_{h}(t), \boldsymbol{i}_{h}(t)\right), \Pi_{h} \boldsymbol{j}_{h}\right)=0,
\end{gathered}
$$

for all $w_{h} \in V_{h} j_{h} \in\left[V_{h}\right]^{s}$, and associated to the initial data $v_{h}(0)=\gamma_{h} v(0)$, where $\gamma_{h}: H^{1}(\Omega) \rightarrow V_{h}$ is a projection defined by the diffusion operator as follows:

$$
\sum_{j=1}^{N_{h}} w_{h}\left(x_{j}\right) \int_{\partial K_{j}^{*}} \nabla\left(v-\gamma_{h} v\right) \cdot \boldsymbol{n}=0, \quad \forall w_{h} \in V_{h} .
$$

Using, for example, $[31,32]$, it is possible to reformulate this problem as a Petrov-Galerkin scheme: For $t>0$, find $\left(v_{h}(t), \boldsymbol{i}_{h}(t)\right) \in\left[V_{h}\right]^{s+1}$ such that

$$
\begin{gathered}
C_{m} \chi^{-1} \int_{\Omega} \partial_{t} v_{h}(t) w_{h} d x+\int_{\Omega} \sigma \nabla v_{h}(t) \cdot \nabla w_{h} d x+\chi^{-1} \int_{\Omega} \mathbf{I}_{\text {ion }}\left(v_{h}(t), i_{h}(t)\right) \pi_{h} w_{h} d x=\int_{\Omega} \mathbf{I}_{\mathrm{app}} \pi_{h} w_{h} d x \quad \forall \pi_{h} w_{h} \in V_{h}^{*}, \\
\int_{\Omega} \mathrm{d}_{t} \boldsymbol{i}_{h}(t) \cdot \Pi_{h} \boldsymbol{j}_{h} d x-\int_{\Omega} m\left(v_{h}(t), \boldsymbol{i}_{h}(t)\right) \cdot \Pi_{h} \boldsymbol{j}_{h} d x=0 \quad \forall \Pi_{h} \boldsymbol{j}_{h} \in\left[V_{h}^{*}\right]^{s} .
\end{gathered}
$$

\subsection{Time integration and operator splitting}

The time interval $(0, T)$ is discretized into $N_{t}$ subintervals of length $\Delta t=T / N_{t}$. We will denote with a superscript $n \leq N_{t}$ the quantities associated to a time instant $t^{n}=n \Delta t$. At each time step, the ionic current $\mathbf{I}_{\text {ion }}$ at each $x \in \Omega$ is approximated by the so-called ionic current interpolation (see, e.g., [18]), where the nodal values of the electric current are interpolated linearly onto the quadrature point, that is,

$$
\mathbf{I}_{\text {ion } i}=\sum_{j=1}^{N_{h}} \mathbf{I}_{\text {ion }}\left(v_{j}, i_{j}\right) \psi_{i}(x)
$$


where $v_{i}, i_{i}$ again denote the value of the potential and ionic concentrations at the node $i$ of $\mathcal{T}_{h}$. By doing so, the assembly of the RHS is very efficient. A further gain in efficiency is obtained with the FVE formulation (3.1), because in that case, we have a ionic current interpolation of the form

$$
\tilde{\mathbf{I}_{\text {ion } i}}=\sum_{j=1}^{N_{h}} \mathbf{l}_{\text {ion }}\left(v_{j}, i_{j}\right) \chi_{i}(x) \text {. }
$$

For sake of both performance and accuracy in recovering the wave front, we split the monodomain equations solving first the pure reaction part of the cell model consisting on a set of nodal ODEs, followed by the (time-dependent) diffusive step (see, e.g., [41]). The way that the time derivatives are approximated in each step of this splitting has an important effect on the accuracy of the final solution. For the reaction, part we employ an explicit Euler scheme for the time integration, whereas the diffusion part of the splitting is discretized in time with a second order backward differentiation formula (BDF2, see, e.g., [56]). These considerations will be explored in Section 4. For $\mathbf{V}^{n}, \mathbf{J}^{n}$ known, we compute $\mathbf{V}^{n+1}, \mathbf{J}^{n+1}$ in three steps, using the intermediate variables $\mathbf{V}^{n+\star}, \mathbf{V}^{n+\star \star}, \mathbf{I}_{\text {ion }}^{n+\star \star}$, from

$$
\begin{aligned}
C_{m} \mathbf{V}^{n+\star} & =C_{m} \mathbf{V}^{n}-\Delta t \mathbf{l}_{\text {ion }}^{n} \\
\left(\frac{C_{m} \chi^{-1}}{\Delta t} 3 \mathbf{M}+2 \mathbf{A}\right) \mathbf{v}^{n+\star \star} & =\frac{C_{m} \chi^{-1}}{\Delta t} \mathbf{M}\left(4 \mathbf{V}^{n+\star}-\mathbf{V}^{n}\right), \\
C_{m} \mathbf{V}^{n+1} & =C_{m} \mathbf{V}^{n+\star \star}-\Delta t \mathbf{l}_{\text {ion }}^{n+\star \star} .
\end{aligned}
$$

Here, the vector $\mathbf{I}_{\text {ion }}$ and the FE mass matrix $\mathbf{M}$ are replaced by $\tilde{\mathbf{I}_{\text {ion }}}$ (3.2) and the FVE mass matrix $\tilde{\mathbf{M}}$ whenever a FVE scheme is employed. In addition, we incorporate mass lumping to the algorithm (the mass matrix is substituted by a diagonal approximation). In some scenarios, this technique could induce numerical artifacts (see the discussion in [57]); however, for the cases studied herein, we found that it does not affect the accuracy of the numerical approximation, also preserved by the FVE discretization. Energy-based stability estimates for coupled and splitting-based FE techniques on the bidomain equations can be found in, for example, [58,59].

\subsection{Pseudo-ECG computation}

The computation of the ECG signal associated to each electrode (2.2) requires the approximation of the function $f_{h}=\Delta v_{h}$ and its integral [60]. Writing formally the following weak form of this relation in $\Omega$ allows us to employ a low order FE approximation $v_{h}$ of $v$ (otherwise, $v_{h}$ should be at least in $V_{h}^{2}=\left\{w_{h} \in C^{0}(\bar{\Omega}):\left.w_{h}\right|_{K} \in \mathbb{P}_{2}(K), \forall K \in \mathcal{T}_{h}\right\}$, where $\mathbb{P}_{2}(K)$ is the space of quadratic polynomials defined on $K$ ):

$$
-\int_{\Omega} \nabla v_{h} \cdot \nabla w_{h} d x=\int_{\Omega} f_{h} w_{h} d x, \quad \forall w_{h} \in V_{h}
$$

Starting from (3.3), if we now assume that $f_{h} \in V_{h}$, then $v_{h}=\sum_{j=1}^{N_{h}} v_{j} \psi_{j}$ and $f_{h}=\sum_{j=1}^{N_{h}} f_{j} \psi_{j}$, which entails that computing $f_{h}$ yields the system

$$
\sum_{j=1}^{N_{h}} f_{j} \int_{\Omega} \psi_{j} \psi_{k} d x=-\sum_{j=1}^{N_{h}} v_{j} \int_{\Omega} \nabla \psi_{j} \cdot \nabla \psi_{k} d x, \quad k=1, \ldots, N_{h}
$$

or in matrix form: Find $\mathbf{f}=\left(f_{1}, \ldots, f_{N_{h}}\right)$ such that

$$
\mathbf{M f}=\hat{\mathbf{A}} \mathbf{V},
$$

where $\hat{\mathbf{A}}$ has elements $\hat{a}_{k l}:=\sum_{K \in \mathcal{T}_{h}} \int_{K} \nabla \psi_{k} \cdot \nabla \psi_{l} d x$. Then, (2.2) is approximated by the quantity

$$
\operatorname{ECG}_{h}\left(x_{e}\right)=\sum_{j=1}^{N_{h}} \frac{f_{j}}{r_{j}}
$$

where $r_{j}:=\left\|x_{j}-x_{e}\right\|$ is the distance from any mesh point to the electrode (located evidently outside $\Omega$ ).

\section{Numerical results}

The computational domain consists of a slab of $1 \times 1 \times 0.0625 \mathrm{~cm}$. The geometry and a series of meshes were generated using the open source mesh manipulator GMSH [61]. All simulations presented in this section have been implemented in the framework of the LGPL parallel FE library LifeV (http://www.lifev.org) and run on one to eight nodes of the cluster Bellatrix at the EPF Lausanne (each with two Sandy Bridge processors running at $2.2 \mathrm{GHz}$, with eaight cores each, $32 \mathrm{~GB}$ of RAM, Infiniband QDR 2 : 1 connectivity, and GPFS filesystem). All model parameters, unless otherwise specified, are taken as in Table I.

The accuracy of the FE and FVE approximations has been assessed by solving the isotropic monodomain equations with the simpler Rogers-McCulloch kinetics [19], where initial and boundary conditions are imposed such that the exact solution is given by $v(x, y, z, t)=(1+0.001 \exp [\sqrt{1 / 2}(x-t)])^{-1}$. An experimental convergence of $O(h)$ is observed for the transmembrane potential in the $H^{1}$-norm for both discretizations (Figure 2 (right), see also [13]). Further code validation has been performed by focusing initially on the accuracy of the CV under the assumption of tissue isotropy, that is, we put $\sigma_{f}=\sigma_{s}=0.001, \mathrm{~cm}^{2} / \mathrm{ms}$. In principle, the meshsize should be taken small enough to reproduce the correct CV, also for conductivity values given specifically as in Section 2 . We have solved the monodomain equations for a sequence of refined meshes, and as observed in Figure 2 (left), when $h$ decreases, the CV goes asymptotically to the physiological value that agrees with [21], and in particular, a meshsize smaller than $h=0.01 \mathrm{~cm}$ is needed. We 
also see that a first order splitting yields too large CV, and moreover, convergence is not attained. On the other hand, we are able to capture the correct value of CV employing a second order splitting method (Figure 2, right).

Next, we consider the anisotropic case. Fibers and sheetlets directions $\left(\mathbf{f}_{0}, \mathbf{s}_{0}\right)$ have been generated analytically as depicted in Figure 1 and were assigned to each vertex of the corresponding mesh. An initial stimulus is applied at one corner of the domain, and we observe the influence of the pacing location on the shape and speed of propagation of the transmembrane potential. Steep depolarization wavefronts are typically observed, which require high spatial resolutions. Figure 3 shows the differences between the wave propagation in two different scenarios. We observe a more significant diffusion of $v$ along the fibers direction. A local CV of $66.3 \mathrm{~cm} / \mathrm{s}$ has been obtained in the fibers direction and of $31.4 \mathrm{~cm} / \mathrm{s}$ in the cross-fibers direction. The ratio between these values is in accordance with the theoretical one $\sqrt{\sigma_{s} / \sigma_{f}}$ given in [62].

We now turn to the analysis of restitution curves, obtained by plotting the APD versus the duration of the previous diastolic interval (DI). These data are generated via a pacing down protocol (see, e.g., [53]), where starting from a first cycle length (CL), defining a reduction factor $\delta t$ and a number of $\ell$ stimulations (typically depending on the complexity of the ionic model at hand), we pace $\ell$ times at a fixed $C L$ and then reduce the pacing interval by $\delta$. This procedure is applied until a fixed minimal $C L$ is reached, where or capture is no longer possible or fibrillation arises. For each pacing frequency, we record the last two APDs and DIs (if no alternans appear, only the last values are needed). These values are computed at fixed locations, far enough from the pacing site. In this case, we employ $\ell=5$ and $\delta t=5 \mathrm{~ms}$. The stimuli are applied at a corner of the domain generating spherical waves propagating across the tissue. For the isotropic case, the data of interest are recorded at several points, far from the stimulus location and in the direction of the propagation (i.e., along the diagonal of the $x y$-plane). For the anisotropic case, the restitution curves and recording locations are depicted in Figure 4. We observe that irrespective of the location, the shape of the restitution curves is conserved, whereas the stable APD (represented by the corresponding visible threshold) varies across the domain.

Pseudo-ECG signals have been obtained from solving (3.4). A block-GMRES solver has been used combined with right ML preconditioner with a tolerance of $10^{-10}$, a maximum of 200 iterations, and presmoothing with symmetric Gauss-Seidel (see, e.g., [63]). An Amesos-KLU solver is employed for the coarse level, with an aggregation threshold of 1\% [64]. Figure 5 shows pseudo-ECGs corresponding to fixed pacing $\mathrm{CL}$ simulations without alternans, performed on a 1D cable, a 2D surface, and the 3D slab. In all cases, a satisfactory
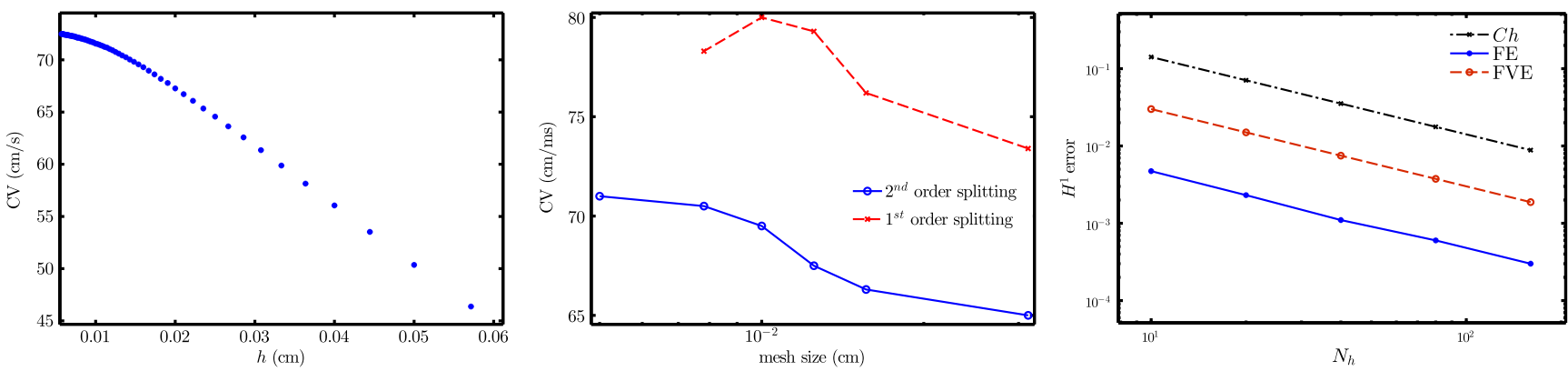

Figure 2. Conduction velocity versus meshsize on a cable computed with the minimal model (left), comparison of conduction velocities computed with FE and first or second order splittings of the minimal model in a 3D slab (middle), and spatial convergence of the FVE and FE approximations of the transmembrane potential at $t=1 \mathrm{~ms}$, with respect to an exact solution employing Rogers-McCulloch kinetics (right).
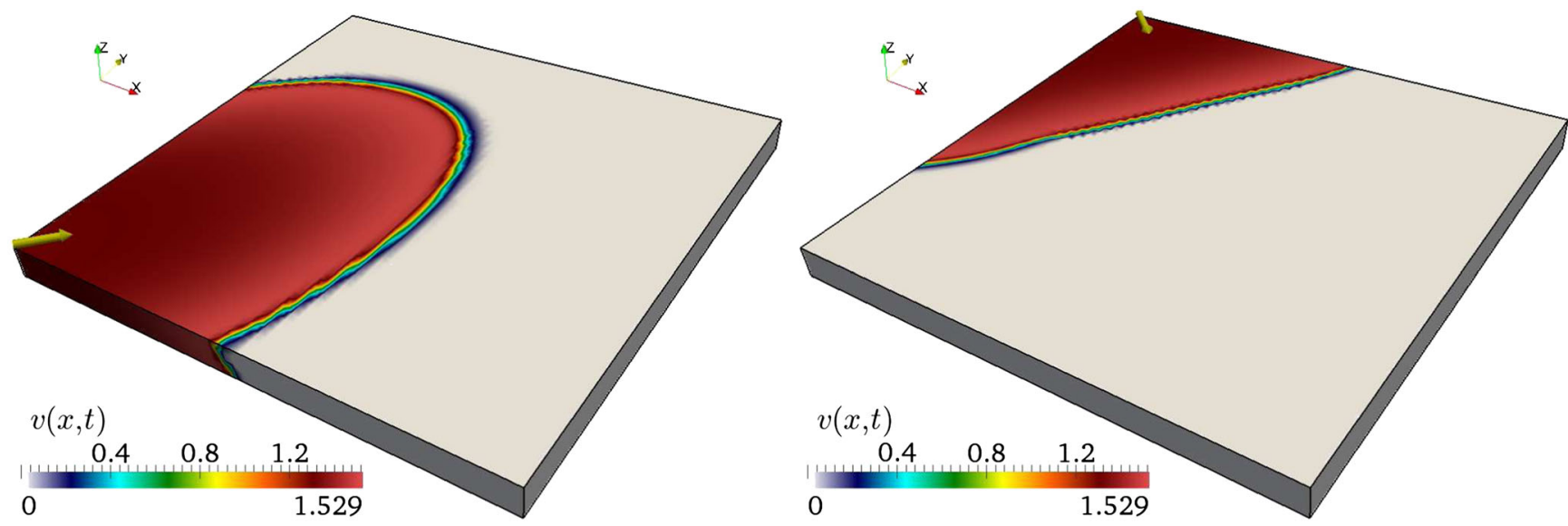

Figure 3. FVE approximation of the transmembrane potential at $t=12 \mathrm{~ms}$. Here, we focus on the effect of anisotropy and pacing location (arrow) on the propagation of the action potential at $t=12 \mathrm{~ms}$, pacing from $(0,0,0)$ (left), and pacing from $(0,1,0)$ (right) for the minimal model. 

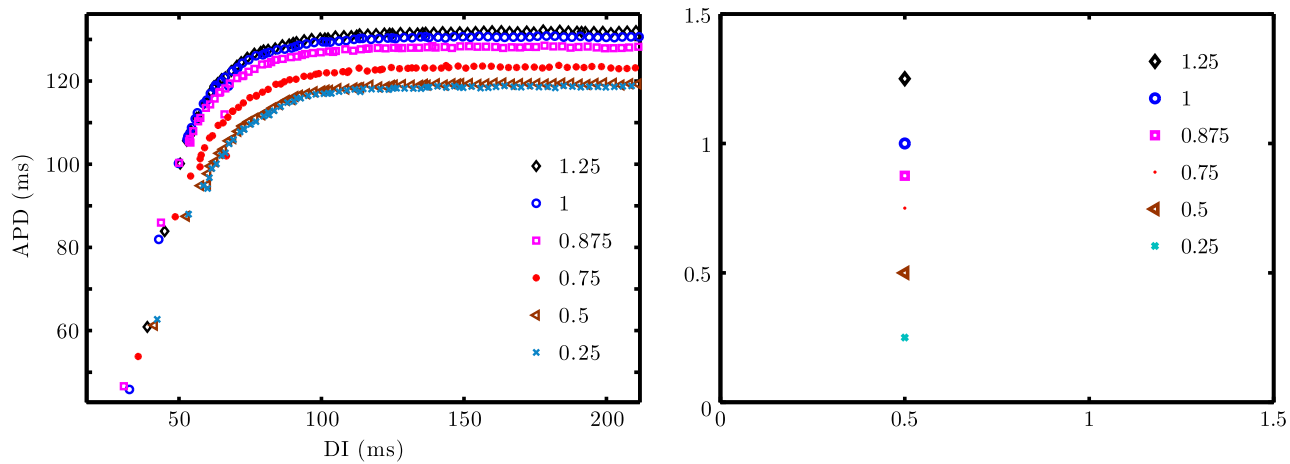

Figure 4. Restitution curves for the minimal model (left) along with recording locations within a $2 \mathrm{D}$ slice of anisotropic tissue (right).
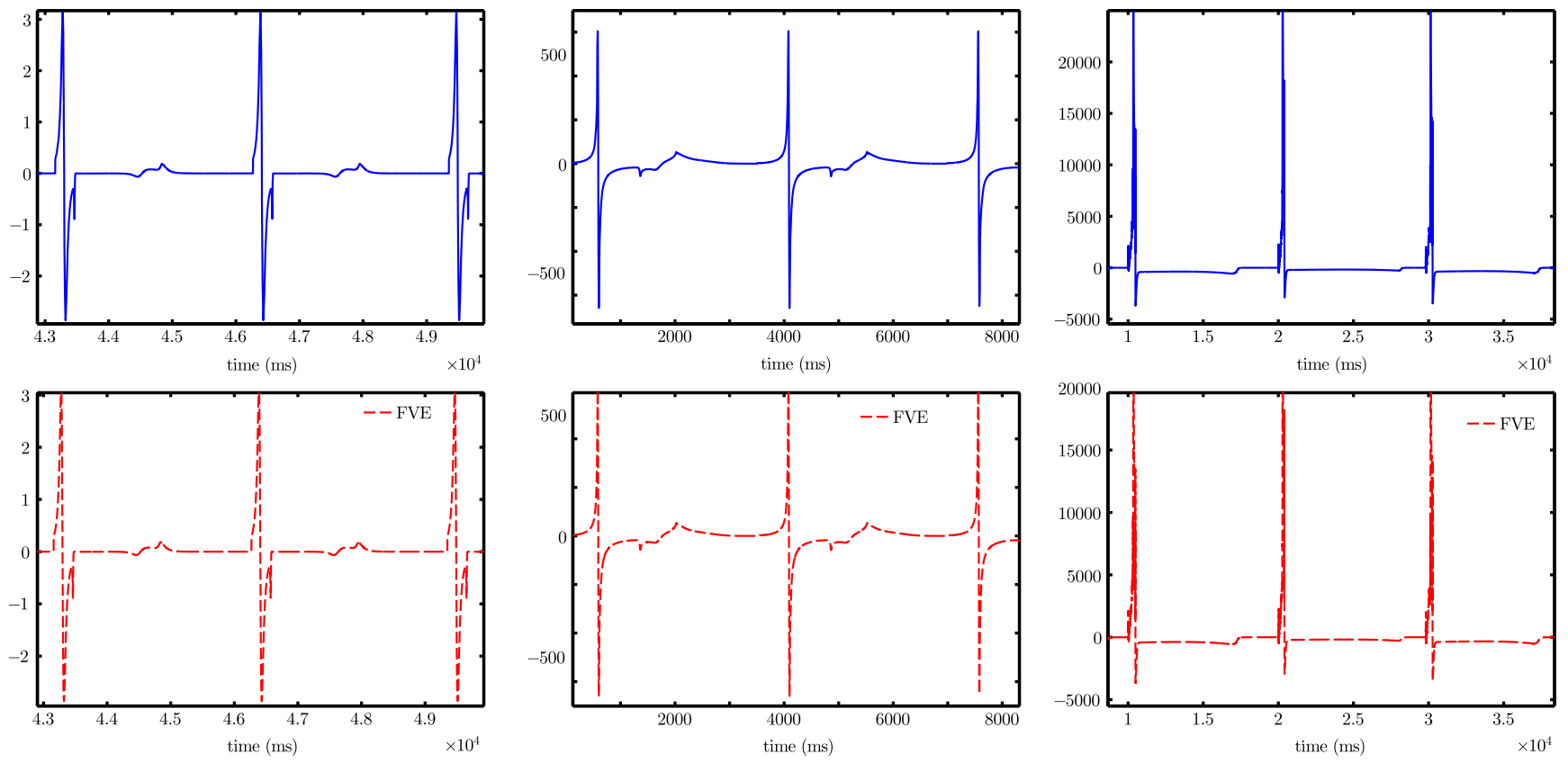

Figure 5. Pseudo-ECG profiles for the minimal model computed on a cable (left), a square surface (middle) and a 3D slab (right) using a FE (top, solid blue) and a FVE space discretization (bottom, dashed red).
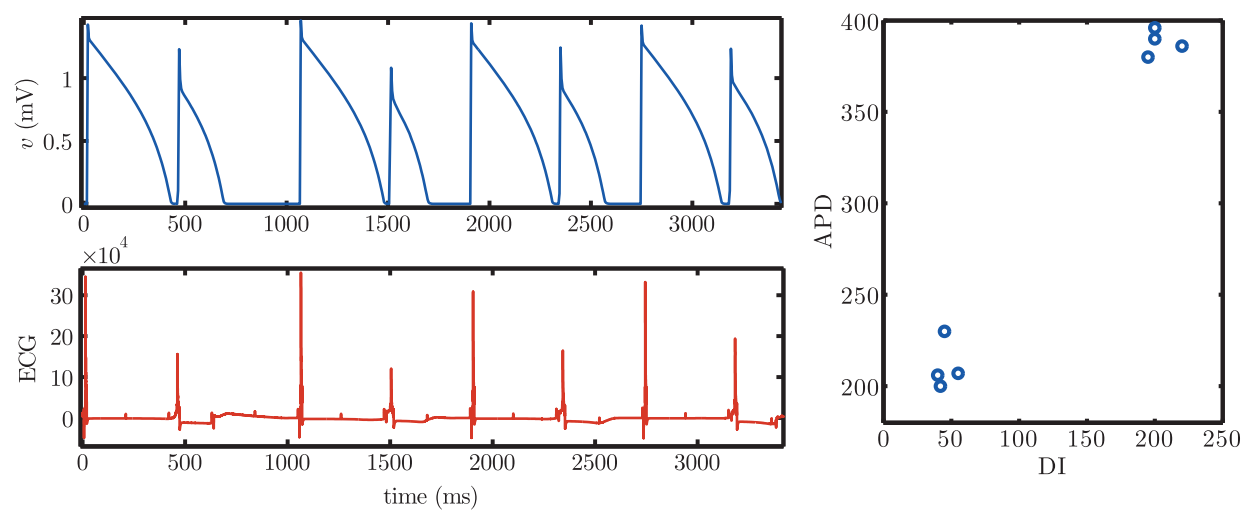

Figure 6. Action potential and related pseudo-ECG signal for the minimal model under isotropic conditions (left) along with local restitution data for an effective cycle length of $420 \mathrm{~ms}$ (right). 

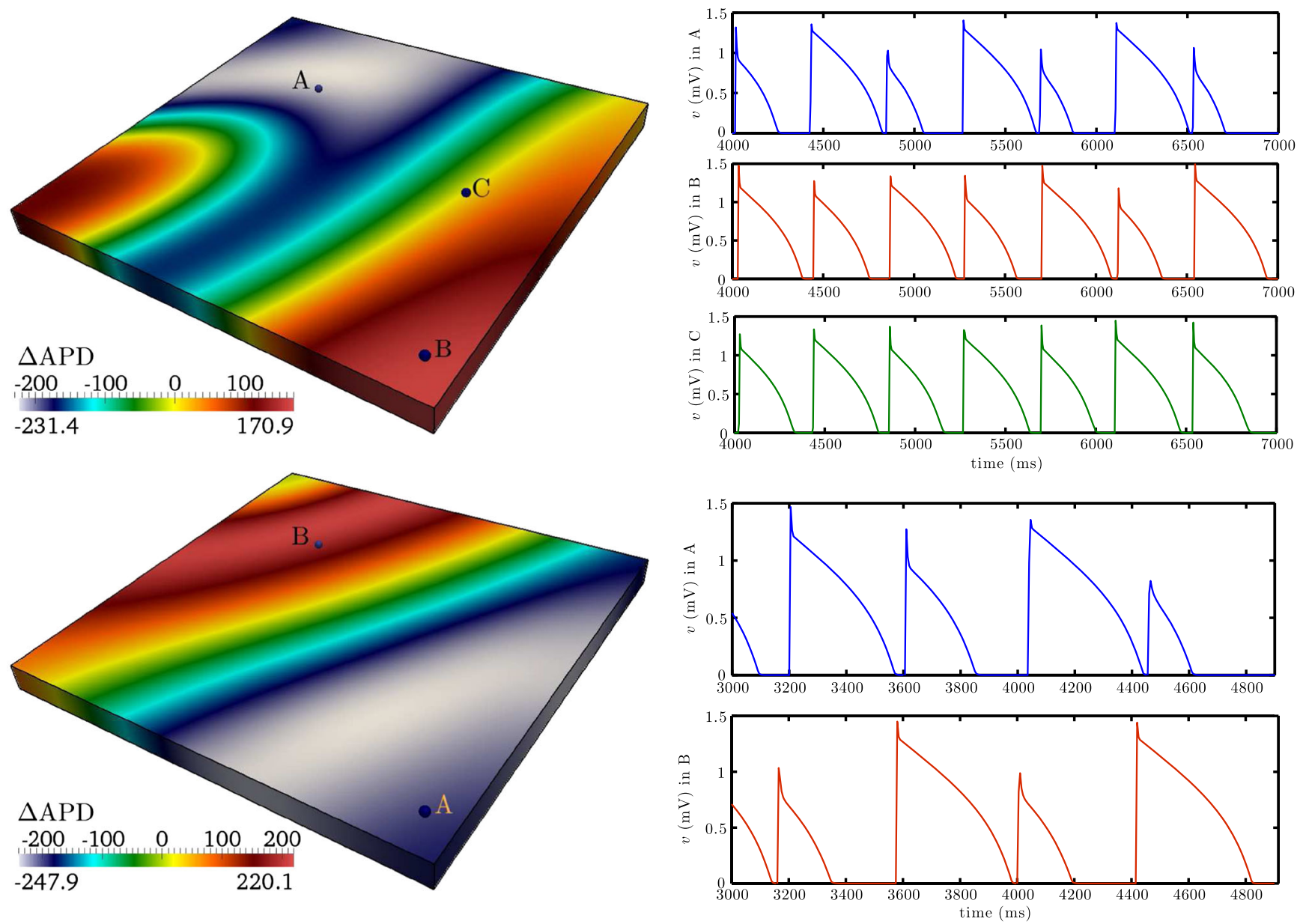

Figure 7. FE approximate spatial distribution of $\triangle \mathrm{APD}$ exhibiting discordant alternans at $t=7000 \mathrm{~ms}$ and $t=5000 \mathrm{~ms}$ (left-top and left-bottom, respectively) and action potential recordings at different locations obtained by pacing at $(0,0,0)$ (right-top) and $(0,1,0)$ (right-bottom).

qualitative agreement with respect to the expected structure of the signal is observed. In particular, the QRS complex and T-wave are reproduced according to physiological regimes [41]. The differences between FE and FVE formulations are barely noticeable in this case.

Finally, we describe the dynamics of cardiac alternans as defined in (2.3). It is well known that high pacing frequencies may generate APD alternans (see, e.g., [53] and references therein). We here employ a so-called quiescent pacing protocol, where from a resting initial condition, we immediately start pacing at the desired frequency. We observe that a pacing period of $210 \mathrm{~ms}$ already induces alternation of the APD. Only one of two action potentials is kept, and therefore, the effective cycle length is of $420 \mathrm{~ms}$.

Under the assumption of tissue isotropy, we observe an homogeneous spatial distribution for the generated alternans, which are spatially concordant (Figure 6, left-top). The presence of alternans is also noticed from Figure 6 (right), where the restitution curve related to this specific $\mathrm{CL}$ exhibits a splitting (long-short repetition). As predicted, we observe a correlation between APD alternans and T-wave alternans (Figure 6, left), and an alternation in the amplitude of the action potential is correlated with alternation of the QRS complex amplitude in the pseudo-ECG counterpart.

We now take into account the tissue anisotropy and pace from two different locations $(0,0,0)$ and $(0,1,0)$. As in the previous case, only one out of two action potentials is kept. The resulting spatial distribution of transient alternans is no longer homogeneous and spatially discordant alternans are observed. In addition, the fibers direction has an important effect in the alternans distribution. From Figure 7, we see that both the shape of the action potential and the alternans pattern depend on the pacing site too. At the recording locations $A, B$, we observe out-of-phase alternans (discordant alternans), whereas at $C$, we observe a nodal line, though the pacing location is surrounded by an in-phase alternans pattern.

We also study the presence of spiral waves in the tissue in both isotropic and anisotropic cases using the proposed FVE method. Because the obtained wavelength is smaller in this case, we employ the Rogers-McCulloch model. As expected, both cases present perturbed pseudo-ECG signals. In the isotropic case, because the spiral wave does not stabilize, the initial form of the signal can be easily identified; however, the QRS complex shows some abnormalities, see Figure 8. In the anisotropic case, the spiral wave reaches a periodic state, and after stabilization, the general form of the signal is completely destroyed. The fact that spiral waves are associated with tachycardia and fibrillation suggests that the pseudo-ECG reproduces correctly the underlying rhythm disorders. 

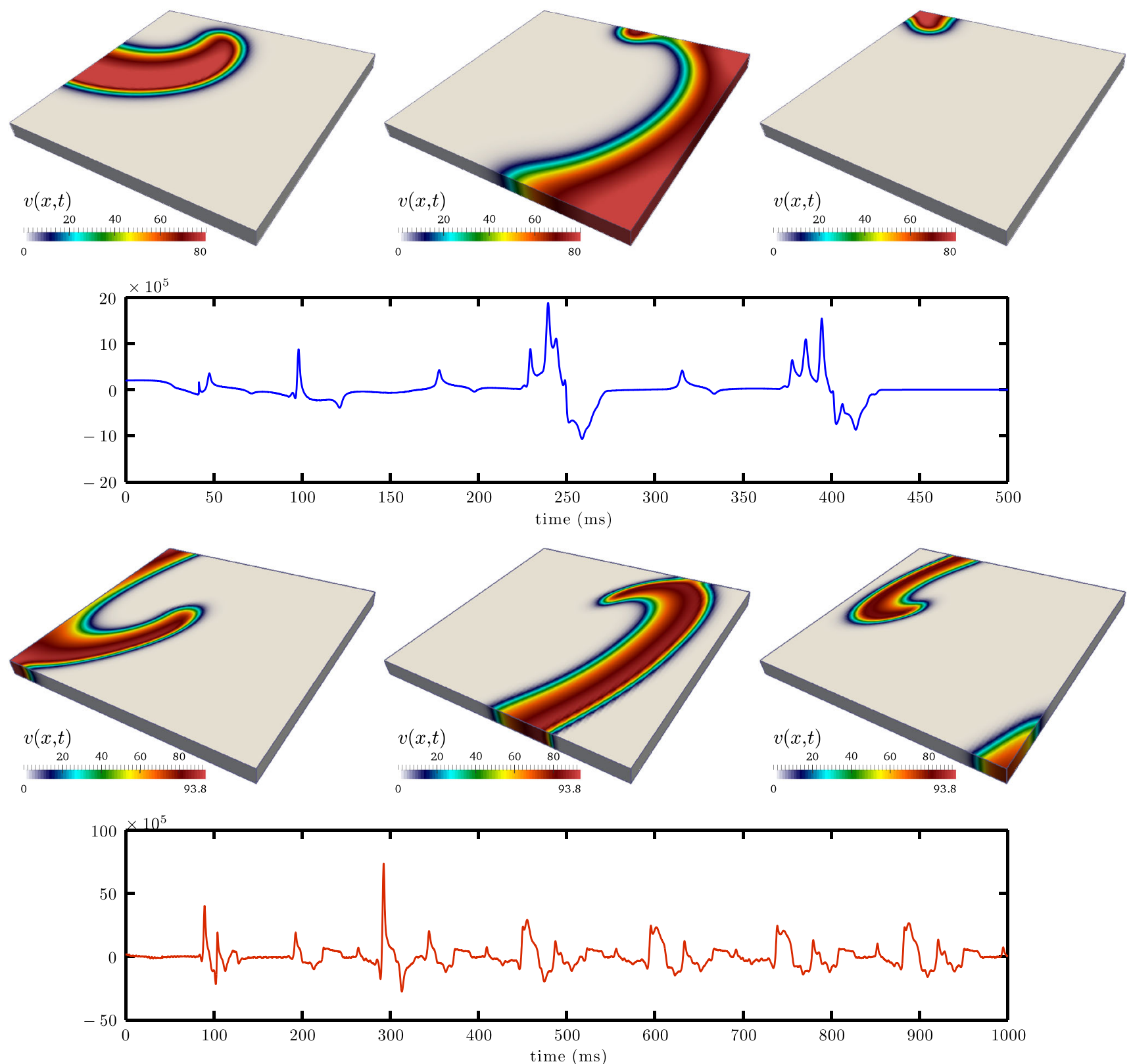

Figure 8. Snapshots of the FVE approximation of the transmembrane potential at several time instants and pseudo-ECG signal reconstructed with the RogersMcCulloch model on isotropic (top) and anisotropic tissue conditions (bottom).

\section{Concluding remarks}

Alternans patterns, action potential repolarization, and spiral waves breakup are often associated with serious pro-arrhythmic states in cardiac tissue. Therefore, understanding the nonlinear spatio-temporal mechanisms underlying these phenomena is of crucial importance for the medical practice. In this paper, we have focused in particular on the development of cardiac alternans exploring a phenomenological mathematical model fine tuned on cardiac electrophysiological data both on 1D, 2D, and 3D idealized domains. A careful validation of the code was performed reproducing correct CV and APD shapes and wavelengths (Figure 2). In the threedimensional case, fibers rotational anisotropy throughout the thickness of the domain was also considered in the model (Figure 3), highlighting that the influence of the pacing location is affected by the fibers architecture in the tissue. In particular, two totally different action potential waves are generated with a net measurable reduction of the propagation velocity when a pacing site orthogonal to the fibers direction is employed.

A specific analysis of the APD restitution curves (Figure 4) allows us to emphasize that the onset of the APD-DI heterogeneities are also due to the presence of fibers in the tissue. Although the shape of the restitution curves is similar at different locations in the domain, the starting point with a slope greater than 1 arises for different value of APD. Such an observation is also in line with the numerical quantification of spatial discordant alternans observed in our three-dimensional simulations (Figure 7). The same observation is further 
reinforced through the analysis of the pseudo-ECG signals. In fact, simulations performed on isotropic (1D, 2D, and 3D) domains and at low pacing frequencies do not show any alternans in the ECG profiles (Figure 5), whereas the same quantities analyzed at higher pacing frequency clearly discriminate an alternating regime (splitting) both in concordant (Figure 6) and discordant (Figure 7) cases by varying the T-wave duration and the QRS amplitude. Finally, we analyzed the profiles of the pseudo-ECG signal in the case of a stable arrhythmia, that is, a single spiral in the tissue (Figure 8). By comparing isotropic and anisotropic three-dimensional slabs, we correctly observe as the last case results in much more realistic ECG signals, very close to a prefibrillating scenario typical of diseased heart tissues.

In addition, from a numerical perspective, our results suggest that, at least for the minimal model, a second order splitting combined with mass lumping is an accurate enough choice to recover the correct conduction velocities. However, complex intramural patterns are also present in the behavior of the potential and its corresponding ECG reconstructions. These aspects deserve further investigation.

Current extensions of this work include the study of excitation-contraction phenomena and the assessment of mechanical alternans in cardiomyocytes and in the whole muscle using an active strain framework (see, e.g., [65-67]). A further extension toward thermal and mechanical couplings is also under investigation: in particular, the specific aim is to account for more realistic scenarios in order to validate recent low energy defibrillating techniques [68-71]. Our mathematical framework is in accordance with other recent works addressing the problem of spiral pinning and unpinning in biological excitable and deformable tissues, with particular reference to the heart tissue $[68,72,73]$. Our work represents an extension to these studies, which were typically treating isotropic, two-dimensional domains with over-simplified phenomenological models.

The approach here proposed, moreover, allows extensions and applications for related problems in biological excitable media, that is, intestine [74-76] and brain [77, 78] as well as other biochemical systems [79]. The robustness of the pseudo-ECG calculation in such a complex phenomenon is quite appealing and promising and will be further explored in combination with advanced numerical techniques of signal analysis. The introduction of innovative and predictive synthetic indicators (see, e.g., [80]) for the clinical practice will be also carefully considered in a forthcoming contribution.

We also plan to carry out the convergence analysis of FVE formulations for monodomain and bidomain equations following [31]. Further improvements of this work, more oriented to numerical methods, may include the assessment of the influence of space adaptivity in cardiac electrophysiology [4] and electromechanics, and the development of efficient and scalable preconditioners that would allow an increased performance $[17,81]$.

\section{Acknowledgements}

The authors acknowledge the financial support by the European Research Council through the Advanced Grant Mathcard, Mathematical Modelling and Simulation of the Cardiovascular System, Project 227058 and the International Center for Relativistic Astrophysics Network, ICRANet. Fruitful discussions with Simone Rossi (EPF Lausanne) are also gratefully acknowledged.

\section{References}

1. Winfree AT. When Time Breaks Down: The Three-Dimensional Dynamics of Electrochemical Waves and Cardiac Arrhythmias. Princeton University Press: Princeton, 1987.

2. Bini D, Cherubini C, Filippi S, Gizzi A, Ricci PE. On spiral waves arising in natural systems. Communications in Computational Physics 2010; 8:610-622.

3. Cherry EM, Fenton FH. Visualization of spiral and scroll waves in simulated and experimental cardiac tissue. New Journal of Physics 2008; 10:883-911.

4. Deuflhard P, Erdmann B, Roitzsch R, Lines GR. Adaptive finite element simulation of ventricular fibrillation dynamics. Physical Review E: Statistical, Nonlinear, and Soft Matter Physics 2012; 85:031915.

5. Luengviriya C, Luengviriya J, Sutthiopad M, Müller SC. Influence of temperature on a spiral wave in excitable chemical media. IPCBEE 2012; 38: 105-109.

6. Fenton FH, Gizzi A, Cherubini C, Pomella N, Filippi S. Role of temperature on nonlinear cardiac dynamics. Physical Review E: Statistical, Nonlinear, and Soft Matter Physics 2013; 042709:87.

7. Panfilov AV, Holden AV (eds). Computational Biology of the Heart eds. Wiley: Chichester, 1997.

8. Hodgkin AL, Huxley AF. A quantitative description of membrane current and its application to conduction and excitation in nerve. Journal of Physiology 1952; 117:500-544.

9. Cherubini C, Filippi S. Lagrangian field theory of reaction-diffusion. Physical Review E: Statistical, Nonlinear, and Soft Matter Physics 2009; 80:046117.

10. Bordas R, Carpentieri B, Fotia G, Maggio F, Nobes R, Pitt-Francis J, Southern J. Simulation of cardiac electrophysiology on next-generation high-performance computers. Philosophical Transactions of the Royal Society of London A 2009; 367:1951-1969.

11. Heidenreich EA, Rodriguez JF, Gaspar FJ, Doblaré M. Fourth-order compact schemes with adaptive time step for monodomain reaction-diffusion equations. Journal of Computational Physics 2008; 216:39-55.

12. Skouibine K, Trayanova N, Moore P. A numerically efficient model for simulation of defibrillation in an active bidomain sheet of myocardium. Mathematical Biosciences 2000; 166:85-100.

13. Bendahmane M, Bürger R, Ruiz-Baier R. A finite volume scheme for cardiac propagation in media with isotropic conductivities. Mathematics and Computers in Simulation 2010; 80:1821-1840.

14. Harrild DM, Henriquez CS. A finite volume model of cardiac propagation. Annals of Biomedical Engineering 1997; 25:315-334.

15. Aliev RR, Panfilov AV. A simple two-variable model of cardiac excitation. Chaos Solitons \& Fractals 1996; 7:293-301.

16. Bourgault $Y$, Ethier M, LeBlanc VG. Simulation of electrophysiological waves with an unstructured finite element method. ESAIM: Mathematical Modelling and Numerical Analysis 2003; 37:649-661.

17. Colli Franzone P, Pavarino LF. A parallel solver for reaction-diffusion systems in computational electro-cardiology. Mathematical Models and Methods in Applied Sciences 2004; 14:883-911. 
18. Pathmanathan P, Murams GR, Southern J, Whiteley JP. The significant effect if the choice of ionic current integration method in cardiac electro-physiological simulations. International Journal of Numerical Methods in Biomedical Engineering 2011; 27:1751-1770.

19. Rogers JM, McCulloch AD. A collocation-Galerkin finite element model of cardiac action potential propagation. IEEE Transactions on Biomedical Engineering 1994; 41:743-757.

20. Arthurs CJ, Bishop MJ, Kay D. Efficient simulation of cardiac electrical propagation using high order finite elements. Journal of Computational Physics 2012; 231:3946-3962.

21. Bueno-Orovio A, Cherry EM, Fenton FH. Minimal model for human ventricular action potential in tissue. Journal of Theoretical Biology 2008; 253: 544-560.

22. Iyer V, Mazhari R, Winslow RL. A computational model of the human left-ventricular epicardial myocyte. Biophysical Journal 2004; 87:1507-1525.

23. Bürger R, Ruiz-Baier R. Torres H. A stabilized finite volume element formulation for sedimentation-consolidation processes. SIAM Journal of Scientific Computing 2012; 34:B265-B289.

24. Cai Z. On the finite volume element method. Numerische Mathematik 1991; 58:713-735.

25. Ewing RE, Lazarov RD, Lin Y. Finite volume element approximations of nonlocal reactive flows in porous media. Numerical Methods for Partial Differential Equations 2000; 16:285-311.

26. Girault V. A combined finite element and marker and cell method for solving Navier-Stokes equations. Numerische Mathematik 1976; 26:39-59.

27. Quarteroni A, Ruiz-Baier R. Analysis of a finite volume element method for the Stokes problem. Numerische Mathematik 2011; 118:737-764.

28. Ruiz-Baier R, Torres $\mathrm{H}$. Numerical solution of a multidimensional sedimentation problem using finite volume-element methods. Applied Numerical Mathematics 2014, DOI 10.1016/j.apnum.2013.12.006.

29. Penland RC, Harrild DM, Henriquez CS. Modeling impulse propagation and extracellular potential distributions in anisotropic cardiac tissue using a finite volume element discretization 2002; 4:215-226.

30. Bürger R, Ruiz-Baier R, Tian C. Stability analysis and finite volume element discretization for delay-driven spatial patterns in a predator-prey model. Journal of Mathematical Analysis and Applications. submitted 2013.

31. Lazarov R, Tomov S. A posteriori error estimates for finite volume element approximations of convection-diffusion-reaction equations. Computers \& Geosciences 2002; 6:483-503.

32. Lin Z, Ruiz-Baier R, Tian C. Finite volume element approximation of an inhomogeneous Brusselator model with cross-diffusion. Journal of Computational Physics 2014; 256:806-823.

33. Phongthanapanich $S$, Dechaumphai P. Finite volume element method for analysis of unsteady reaction-diffusion problems. Acta Mechanica Sinica 2009; 25:481-489.

34. Sun J, Amellal F, Glass L, Billette J. Alternans and period-doubling bifurcations in atrioventricular nodal conduction. Journal of Theoretical Biology 1995; 173:79-91.

35. Courtemanche M, Glass L, Keener JP. Instabilities of a propagating pulse in a ring of excitable media. Physical Review Letters 1993; 70:2182-2185.

36. Ito H, Glass L. Theory of reentrant excitation in a ring of cardiac tissue. Physica D 1992; 56:84-106.

37. Nolasco JB, Dahlen RW. A graphic method for the study of alternation of cardiac action potentials. Journal of Applied Physiology 1968; 25: 191-196.

38. Kocica MJ, Corno AF, Carreras-Costa F, Ballester-Rodes M, Moghbel MC, Cueva CN, Lackovic V, Kanjuh VI, Torrent-Guasp F. The helical ventricular myocardial band: global, three-dimensional, functional architecture of the ventricular myocardium. European Journal of Cardio-Thoracic Surgery 2006; 1:S21-40.

39. Pullan AJ, Buist ML, Cheng LK. Mathematically Modeling the Electrical Activity of the Heart: From Cell to Body Surface and Back. World Scientific Publishing Company: Singapore, 2005.

40. Veneroni M. Reaction-diffusion systems for the microscopic cellular model of the cardiac electric field. Mathematics/ Methods in the Applied Sciences 2006; 29:1631-1661.

41. Sundnes J, Lines GT, Tveito A. An operator splitting method for solving the bidomain equations coupled to a volume conductor model for the torso. Mathematical Biosciences 2005; 194:233-248.

42. Boulakia M, Cazeau S, Fernández MA, Gerbeau JF, Zemzemi N. Mathematical modeling of electrocardiograms: a numerical study. Annals of Biomedical Engineering 2010; 38:1071-1097.

43. Aslanidi OV, Clayton RH, Lambert JL, Holden AV. Dynamical and cellular electrophysiological mechanisms of ECG changes during ischaemia. Journal of Theoretical Biology 2005; 237:369-381.

44. Rosenbaum DS, Jackson LE, Smith JM, Garan H, Ruskin JN, Cohen RJ. Electrical alternans and vulnerability to ventricular arrhythmias 1994; 330: 235-241.

45. Pastore JM, Girouard SD, Laurita KR, Akar FG, Rosenbaum DS. Mechanism linking T-wave alternans to the genesis of cardiac fibrillation. Circulation 1999; 99:1385-139.

46. Watanabe MA, Fenton FH, Evans SJ, Hastings HM, Karma A. Mechanisms for discordant alternans. Journal of Cardiovascular Electrophysiology 2001; 12:196-206.

47. Qu Z, Garfinkel A, Chen PS, Weiss JN. Mechanisms of discordant alternans and induction of reentry in simulated cardiac tissue. Circulation 2000; 102:1664-1670.

48. Walker ML, Wan X, Kirsch GE, Rosenbaum DS. Hysteresis effect implicates calcium cycling as a mechanism of repolarization alternans. Circulation 2003; 108:2704-2709.

49. Walker ML, Rosenbaum DS. Cellular alternans as mechanism of cardiac arrhythmogenesis. Heart Rhythm 2005; 2:1383-1386.

50. de Diego C, Pai RK, Dave AS, Lynch A, Thu M, Chen F, Xie L H, Weiss JN, Valderrábano M. Spatially discordant alternans in cardiomyocyte monolayers. American Journal of Physiology-Heart and Circulatory Physiology 2008; 294:H1417-H1425.

51. Ziv O, Morales E, Song Y, Peng X, Odening KE, Buxton AE, Karma A, Koren G, Choi BR. Origin of complex behaviour of spatially discordant alternans in a transgenic rabbit model of type 2 long QT syndrome. Journal of Physiology 2009; 587:4661-4680.

52. Weinberg S, Malhotra N, Tung L. Vulnerable windows define susceptibility to alternans and spatial discordance. American Journal of Physiology-Heart and Circulatory Physiology 2010; 298:H1727-H1737.

53. Gizzi A, Cherry EM, Gilmour RF, Luther S, Filippi S, Fenton FH. Effects of pacing site and stimulation history on alternans dynamics and the development of complex spatiotemporal patterns in cardiac tissue. Frontiers in Physiology 2013; 19(4):71.

54. Visweswaran R, Mclntyre SD, Ramkrishnan K, Zhao X, Tolkacheva EG. Spatiotemporal evolution and prediction of [Ca(2+)]i and APD alternans in isolated rabbit hearts. Journal of Cardiovascular Electrophysiology 2013; 24:1287-95. 
55. Quarteroni A. Numerical Models for Differential Problems, MS\& A series, vol. 2. Springer-Verlag: Milan, 2009.

56. Quarteroni A, Sacco R, Saleri F. Numerical Mathematics, Series: Texts in Applied Mathematics 2nd ed, Vol. 37. Springer-Verlag: Milan, 2007.

57. Pathmanathan P, Bernabeu MO, Niederer SA, Gavaghan DJ, Kay D. Computational modelling of cardiac electrophysiology: explanation of the variability of results from different numerical solvers. International Journal of Numerical Methods in Biomedical Engineering 2012; 28:890-903.

58. Ethier M, Bourgault Y. Semi-implicit time-discretization schemes for the bidomain model. SIAM Journal on Numerical Analysis 2008; 46:2443-2468.

59. Fernández MA, Zemzemi N. Decoupled time-marching schemes in computational cardiac electrophysiology and ECG numerical simulation. Mathematical Biosciences 2010; 226:58-75.

60. Clayton RH, Holden AV. Computational framework for simulating the mechanisms and ECG of re-entrant ventricular fibrillation. Physiological Measurement 2002; 23:707-726.

61. Geuzaine C, Remacle JF. Gmsh: a three-dimensional finite element mesh generator with built-in pre- and post-processing facilities. International Journal for Numerical Methods in Engineering 2009; 79:1309-1331.

62. Fenton $\mathrm{FH}$, Karma A. Vortex dynamics in three-dimensional continuous myocardium with fiber rotation: filament instability and fibrillations. Chaos 1998; 8:20.

63. Sala M. An object-oriented framework for the development of scalable parallel multilevel preconditioners. ACM Transactions on Mathematical Software 2006; 32(3):396-416.

64. Sala M, Stanley K, Heroux M. Amesos: a set of general interfaces to sparse direct solver libraries. Proceedings of PARA'06 Conference, Umea, Sweden, 2006, 976-985.

65. Ruiz-Baier R, Gizzi A, Rossi S, Cherubini C, Laadhari A, Filippi S, Quarteroni A. Mathematical modeling of active contraction in isolated cardiomyocytes. Mathematical Medicine and Biology 2013, DOI 10.1093/imammb/dqt009.

66. Laadhari A, Ruiz-Baier R, Quarteroni A. Fully Eulerian finite element approximation of a fluid-structure interaction problem in cardiac cells. International Journal for Numerical Methods in Engineering 2013; 96:712-738.

67. Nobile F, Quarteroni A, Ruiz-Baier R. An active strain electromechanical model for cardiac tissue. International Journal of Numerical Methods in Biomedical Engineering 2012; 28:52-71.

68. Cherubini C, Filippi S, Gizzi A. Electroelastic unpinning of rotating vortices in biological excitable media. Physical Review E: Statistical, Nonlinear, and Soft Matter Physics 2012; 85:031915.

69. Cherubini C, Filippi S, Nardinocchi P, Teresi L. An electromechanical model of cardiac tissue: constitutive issues and electrophysiological effects. Progress in Biophysics and Molecular biology 2008; 97:562-573.

70. Luther S, Fenton FH, Kornreich BG, Squires A, Bittihn P, Hornung D, Zabel M, Flanders J, Gladuli A, Campoy L, Cherry EM, Luther G, Hasenfuss G, Krinsky VI, Pumir A, Gilmour RF Jr, Bodenschatz E. Low-energy control of electrical turbulence in the heart. Nature 2011; 475:235-239.

71. Pumir A, Sinha S, Sridhar S, Argentina M, Hörning M, Filippi S, Cherubini C, Luther S, Krinsky V. Wave-train-induced termination of weakly anchored vortices in excitable media. Physical Review E: Statistical, Nonlinear, and Soft Matter Physics 2010; 81:010901.

72. Hörning M. Termination of pinned vortices by high-frequency wave trains in heartlike excitable media with anisotropic fiber orientation. Physical Review E 2012; 86:031912.

73. Yuan G, Zhang H, Xu A, Wang G. Attractive and repulsive contributions of localized excitability inhomogeneities and elimination of spiral waves in excitable media. Physical Review E 2012; 88:022920.

74. Gizzi A, Cherubini C, Migliori S, Alloni R, Portuesi R, Filippi S. On the electrical intestine turbulence induced by temperature changes. Physical Biology 2010; 7:16011.

75. O'Grady G, Du P, Paskaranandavadivel N, Angeli TR, Lammers WJ, Asirvatham SJ, Windsor JA, Farrugia G, Pullan AJ, Cheng LK. Rapid high-amplitude circumferential slow wave propagation during normal gastric pacemaking and dysrhythmias. Neurogastroenterology and Motility 2012; 24: e299-312.

76. Lammers WJ. Arrhythmias in the gut. Neurogastroenterology and Motility 2013; 25:353-357.

77. Kochs E. Electrophysiological monitoring and mild hypothermia. Journal of Neurosurgical Anesthesiology 1995; 7:222-228.

78. Zaremba J. Hyperthermia in ischemic stroke. Medical Science Monitor 2004; 10:RA148-153.

79. Jiménez ZA, Steinbock $O$. Stationary vortex loops induced by filament interaction and local pinning in a chemical reaction-diffusion system. Physical Review Letters 2012; 109:098301.

80. Gizzi A, Bernaschi M, Bini D, Cherubini C, Filippi S, Melchionna S, Succi S. Three-band decomposition analysis of wall shear stress in pulsatile flows. Physical Review E 2011; 83:031902.

81. Plank G, Liebmann M, Weber dos Santos R, Vigmond EJ, Haase G. Algebraic multigrid preconditioner for the cardiac bidomain model. IEEE Transactions on Biomedical Engineering 2007; 54:584-597. 